\title{
Are Corrupted Non-dysplastic Colonic Crypts the First Histological Event in Experimental Colonic Carcinogenesis?
}

\author{
CARLOS A. RUBIO \\ Gastrointestinal and Liver Research Laboratory, Department of Pathology, \\ Karolinska Institute and University Hospital, Stockholm, Sweden
}

\begin{abstract}
Background: The colonic crypts in rats replicate by symmetric fission at the base of the crypts, and proceeds upwards, to generate two separate identical crypts. Recently, anomalous crypts (called corrupted colonic crypts, CCCs) were found in the colonic mucosa of Sprague-Dawley rats treated with the carcinogen dimethylhydrazine. Here it was investigated whether CCCs develop in the colonic mucosa of another rat strain, treated with a different carcinogen. Materials and Methods: Archived Swiss-roll colon sections from 25 male Fisher-344 rats treated with the mutagen 2amino-6-methyldipyrido imidazole (GLU1) were reviewed. Non-dysplastic and dysplastic CCCs were regarded as those exhibiting asymmetric fission, asymmetric lateral sprouting/lateral fission, basal dilatations, or spatial aberrations of the normal (vertical) axis. Colonic adenomas were found in three out of the 25 specimens. Results: In the entire colonic mucosa of the 25 GLU1-treated rats, 130 nondysplastic CCCs were recorded amongst 357 non-dysplastic crypts with fission (36.4\%). The mean number of nondysplastic CCCs per animal was 5.2 (range=2-12). These numbers only mirror events taking place at a particular time (i.e. at sacrifice). Considering the high cell production rate of the colonic crypts, the actual number of CCCs/rat occurring during the usual mucosal turnover time of 72 hours might be substantial. In the three adenoma specimens, non-dysplastic CCCs were found underneath CCCs with dysplasia. Discussion and Conclusion: For many years, the development of crypt dysplasia and adenoma have been considered the initial histological events in colonic carcinogenesis. This study
\end{abstract}

This article is freely accessible online.

Correspondence to: Carlos A. Rubio, MD, Ph.D., Gastrointestinal and Liver Pathology Research Laboratory, Department of Pathology, Karolinska Institute and University Hospital, 17176, Stockholm, Sweden. Tel: +46 851774527, Fax: +46 851774524, e-mail: Carlos.Rubio@ki.se

Key Words: Colon, rats, crypts, fission, carcinogens. demonstrates that non-dysplastic CCCs also develop in GLU 1-treated Fisher-344 rats. Non-dysplastic CCCs were found underneath CCCs with dysplasia. Non-dysplastic CCCs might act as scaffolds at the time of top-down cell replacement/ transformation of the crypts by dysplastic cells. It is submitted that non-dysplastic CCCs might be the initial histological recordable event in experimental colonic carcinogenesis.

Most colonic carcinomas in rodents evolve in the mucosal domain that occupies the vast majority of that organ (1-3), a domain built with crypts lined by goblet and columnar cells. Carcinomas in this huge mucosal domain are preceded by foci of dysplastic cell proliferations called conventional adenomas (1-3) or serrated adenomas (4). It has been demonstrated that progression to carcinoma is triggered by the accumulation of molecular aberrations (5) and by epigenetic modification of gene expression (6). In the remnant mucosal domain, known as gut-associated lymphoid tissue (GALT), carcinomas induced by dimethylhydrazine (DMH) frequently evolve in Sprague-Dawley (SD) and Fisher 344-rats (7-9).

The normal colon of untreated rats comprises test tubeshaped, close-packed crypts with their axis vertical to the muscularis mисоsa. The colonic crypts replicate by symmetric fission, the division of which begins at the base of the crypts and proceeds upwards until two identical, individual crypts are formed. In a previous study on DMH-treated SD rats, we reported colonic crypts exhibiting anomalous configurations, called corrupted colonic crypts (CCCs) $(10,11)$.

The purpose of this communication was to review archival colonic sections from a previous experiment using another rat strain (Fisher 344; F-344) treated with a different carcinogen [mutagen 2-amino-6-methyldipyrido imidazole (GLU1)] (12-14). The aim was to explore whether CCCs might also evolve in this animal setting.

\section{Materials and Methods}

From a previous experiment (14), archived histological sections from 25 male F-344 rats were retrieved and reviewed. These rats had received pellets containing $500 \mathrm{ppm}$ of the mutagenic pyrolysate compound GLU1, for 24 months. After sacrifice, the 


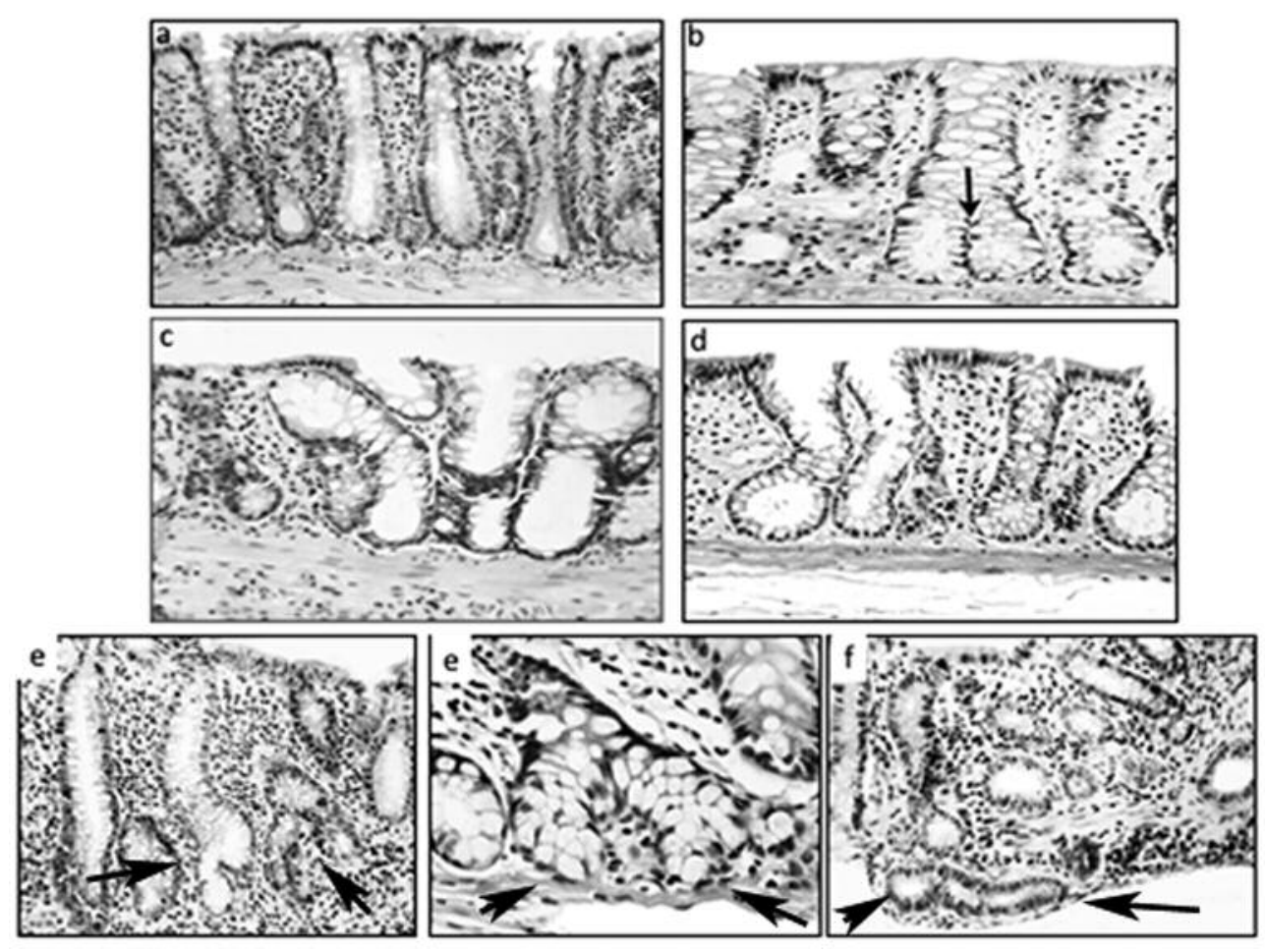

Figure 1. Architecture of the colon in Fisher 344 rats. a: Normal colonic crypts [hematoxylin-eosin (H\&E), $\times 4$ ]. b: Normal colonic crypt with symmetric fission $(H \& E, \times 10)$. c-f: Non-dysplastic colonic corrupted crypts $(H \& E, c: \times 20, b-f: \times 10, c-e: \times 20)$. Note spatial aberration of the normal (vertical) axis at arrows in $f$.

entire colons were processed using the Swiss-roll technique and immediately fixed in formalin.

Non-dysplastic and dysplastic CCCs were regarded those with i) asymmetric fission, ii) asymmetric lateral sprouting, iii) asymmetric lateral fission, iv) basal dilatation ( twice the diameter of the normal lumen or greater), and v) spatial aberrations of the normal (vertical) axis. CCCs were found in groups of consecutive crypts $(\geq 2)$.

Quantification of the crypts with CCCs was performed on entire Swiss-roll histological sections stained with hematoxylin and eosin (H\&E).

Three out of the 25 Swiss-roll sections contained a conventional adenoma.

\section{Results}

The normal colon of untreated F-344 rats comprises test tube-shaped, close-packed crypts with their axis vertical to the muscularis mucosa (15) (Figure 1). The division of a colonic crypt begins at its base and proceeds upwards to form two identical, individual crypts (Figure 1).

In the entire colonic mucosa of the 25 GLU1-treated rats, 130 non-dysplastic CCCs were found amongst 357 nondysplastic crypts with fission (36.4\%). The mean number of non-dysplastic CCCs per animal was 5.2 (range=2-12). In the three adenomas, non-dysplastic CCCs were found underneath CCCs with dysplasia (Figure 2).
Groups of consecutive $(\geq 2)$ crypts were recorded in $15 \%$ of the F-344 rats.

\section{Discussion}

The results of the present survey demonstrate that the colonic mucosa of F-344 rats treated with the mutagen GLU1 develop non-dysplastic CCCs. The most plausible explanation for these findings is that the carcinogen administered induced these architectural crypt aberrations. It may be argued that the number of CCCs per animal was relatively low, namely 5.2 (mean). It should be understood, however, that this number only mirrors events taking place at a particular time (i.e. at sacrifice).

Considering the high cell production rate of colonic crypts, the actual number of $\mathrm{CCC}$ occurring during the mucosal turnover time of 72 hours $(16,17)$ might be substantial.

The CCCs found in GLU1-treated F-344 rats and in DMH-treated SD rats (11) differ from the colonic aberrant crypt foci (ACF) reported by Bird et al. (18) in SD rats treated with methylnitrosourea, inasmuch as colonic ACFs were detected by gross observation of the mucosal surface (18) and not in well-oriented histological sections, as in the 


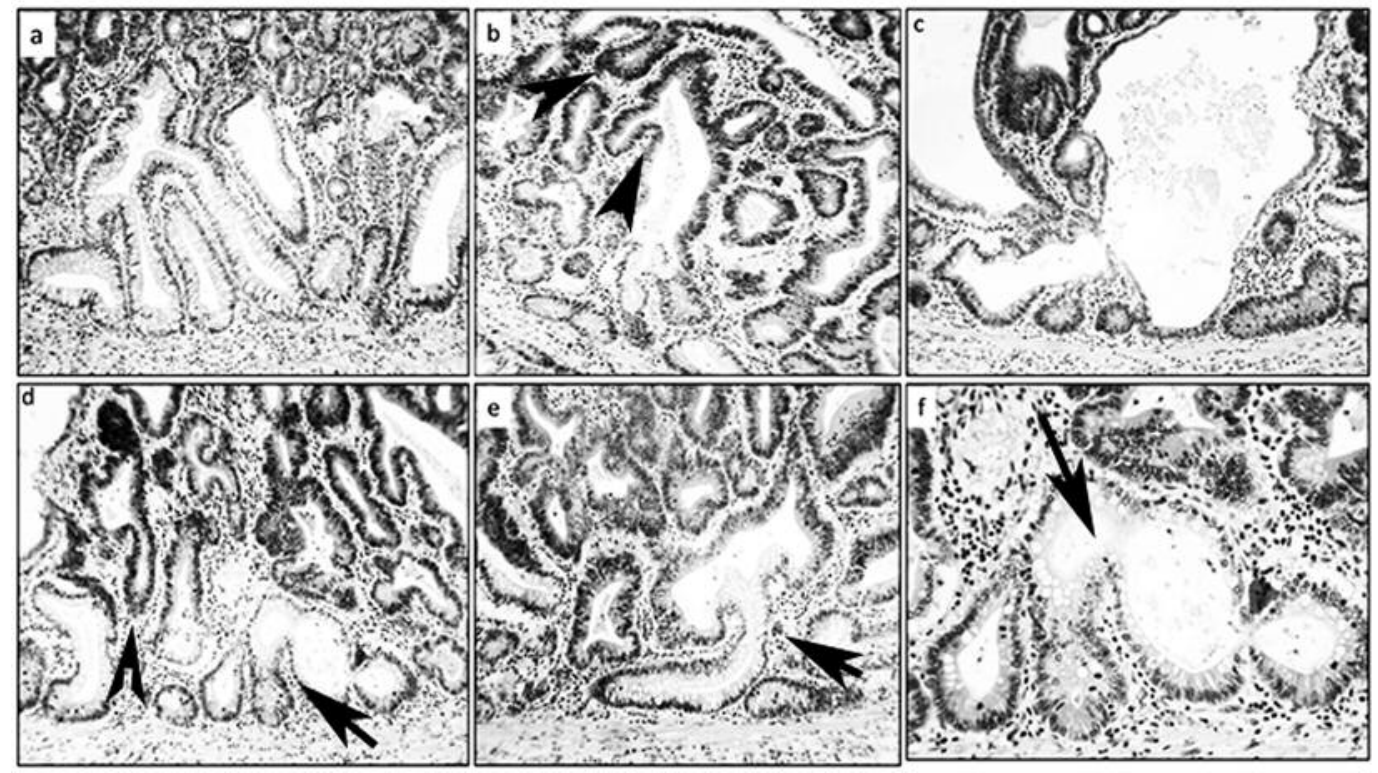

Figure 2. Architecture of the colon mucosa with adenomas in Fisher 344 rats. a-f: Dysplastic (darker) epithelium from conventional colon adenomas (on top), replacing non-dysplastic corrupted colonic crypts underneath (arrows) ( $H \& E \times 20)$.

present study. According to Ghirardi et al. the ACF phenomenon is infrequently found in the distal colon, the site of predilection for carcinogen-induced adenomas and carcinomas (19). Similarly, Ochiai et al. postulated that growth features and distribution of ACFs did not correlate to the distribution of adenomas and carcinomas (20). Here CCCs were predominantly found in the distal colon, the site where crypt dysplasias, adenomas and carcinomas frequently develop in carcinogen-treated rats.

In 1985, St Clair and Osborne isolated colonic crypts from young Holtzman rats by the aid of a microdissection technique (21). Crypts were scored as ordinary or in fission. The percentage of crypts in fission reached peak values of $52 \%$ in the colon at 21-day post-parturition. From this time onwards, the percentage dropped until the adult value of approximately $7 \%$ was reached. During this same period, the number of crypts increased in the colon. Thus, an inverse relationship was found between the percentage of crypt fission and number of crypts. Distribution of fissure heights in fission crypts did not change as the animal aged. The majority of the fissures were found in the lower quarter of the fission crypts, suggesting that as soon as the fissure extends beyond the stem cell zone, division into two crypts occurs (21). More recently, Tan et al. found asymmetrical crypt budding in the colon of C57BL/6 mice (22).

In order to separate colonic crypts from the lamina propria, the muscularis mucosa and the submucosa, the colons were kept and rinsed on ice, incubated in sodium hypochlorite (5 minutes), in EDTA (90 minutes), transferred to phosphate-buffered saline (PBS) $\left(20^{\circ} \mathrm{C}\right)$, shaken vigorously ( 5 times), and centrifuged ( $34 \times g$ for 5 minutes). With this procedure, the isolated crypts (without lamina propria, and muscularis mucosa) exhibited asymetic buddings. It should be pointed out that the aggressive method of Tan et al. (22) differs from the method used here, inasmuch as the colons were immediately fixed in formaldehyde after sacrifice, a technique that precludes possible histological artefacts.

During crypt renewal, the stem cells at the crypt bottom generate amplifying daughter cells that proliferate and differentiate while migrating upwards $(16,17,23)$. Winglessrelated integration site (WNT) signaling is high at the bottom of the crypt, where stem cells reside, and low at the top. In contrast, adenomatous polyposis coli $(A p c)$ gene expression is low at the crypt bottom and high at the top, the domain of differentiated cells $(17,23)$. The $A p c$ gene normally downregulates WNT signaling. Hence, WNT and APC gradients are important in crypt formation and regulation. Since both APC and WNT signaling components are required for mitosis, a zone emerges in the lower crypt where conditions are optimal for maximal cell division and mitosis orientation, resulting in symmetric crypt fission. Boman and Fields recently found that $A P C$ mutation-induced changes in the countercurrent-like mechanism triggered expansion of the proliferative populations and crypt fission (23). These authors postulated that crypts began to show abnormalities in histology only when they became dysplastic, and that the proliferative shift in normal-appearing crypts, not yet 
dysplastic was the earliest-known biological alteration (23). Contrary to those assertions, non-dysplastic CCCs were found in the present study as the first histological alteration in the colonic mucosa of GLU1-treated F-344 rats. Similar findings were recently reported in DMH-treated SD rats (11). Rationally, the non-dysplastic CCCs might had been tailored by $A p c$ mutations generated by the mutagen GLU1 (12-14) and previously by the carcinogen DMH in SD rats (11).

Non-dysplastic CCCs might act as scaffolds at the time of top-down cell replacement/transformation of the crypts by dysplastic cells. It is submitted that non-dysplastic CCCs might be the initial histological recordable event in experimental colonic carcinogenesis.

\section{Acknowledgements}

The Author is indebted to Dr. Shozo Takayama, President of the Princess Takamatsu Cancer Research Fund, Tokyo, Japan, for permitting review, once again, of his experiments on GLU1-treated F344 rats.

\section{References}

1 Druckrey H, Preussmann R, Matzkies F and Ivankovic S: Selective production of intestinal cancer in rats by 1,2dimethylhydrazine. Naturwissenschaften 54: 285-286, 1967.

2 Boivin GP, Washington K, Yang K, Ward JM, Pretlow TP, Russell R, Besselsen DG, Godfrey VL, Doetschman T, Dove WF, Pitot HC, Halberg RB, Itzkowitz SH, Groden J and Coffey RJ: Pathology of mouse models of intestinal cancer: consensus report and recommendations. Gastroenterology 124: 762-77, 2003.

3 Shetye J and Rubio CA: The chronological appearance of flat colonic neoplasias in rats. In Vivo 18: 197-202, 2004.

4 Rubio CA: Traditional serrated adenomas and serrated carcinomas in carcinogen-treated rats. J Clin Pathol 2016 Doi:10.1136/jclinpath-2016-204037.

5 Femia AP, Luceri C, Toti S, Giannini A, Dolara P and Caderni $\mathrm{G}$ : Gene expression profile and genomic alterations in colonic tumours induced by 1,2-dimethylhydrazine (DMH) in rats. BMC Cancer 10: 194-198. 2010.

6 Jang H, Mason JB, and Choi SW: Genetic and epigenetic interactions between folate and aging in carcinogenesis. J Nutr 135: 2967S-2971S, 2000.

7 Deasy JM, Steele G Jr., Ross DS, Lahey SJ, Wilson RE and Madara J: Gut-associated lymphoid tissue and dimethylhydrazine-induced colorectal carcinoma in the Wistar/Furth rat. J Surg Oncol 24: 36-40, 1983.

8 Rubio CA: Lymphoid tissue-associated colonic adenocarcinomas in rats. In Vivo 1: 61-64, 1987.

9 Rubio CA: Three pathways of colonic carcinogenesis in rats. Anticancer Res 37: 15-20, 2017.

10 Rubio CA: The histogenesis of the third pathway of colonic carcinogenesis in rats. Anticancer Res 37: 1039-1042, 2017.
11 Rubio CA: Corrupted colonic crypt fission in carcinogen-treated rats. PLoS One 12: e0172824. Doi: 10.1371, 2017.

12 Ohgaki H, Hasegawa H, Kato T, Suenaga M, Ubukata M, Sato $\mathrm{S}$, Takayama $\mathrm{S}$ and Sugimura T: Carcinogenicity in mice and rats of heterocyclic amines in cooked foods. Environ Health Perspect 67: 129-134, 1986.

13 Takayama S, Masuda M and Mogami M: Induction of cancers in the intestine, liver and various other organs of rats by feeding mutagens from glutamic acid pyrolysate. Jpn J Cancer Res 75: 207-213, 1984.

14 Rubio CA and Takayama S: Difference in histology and size in colonic tumors of rats receiving two different carcinogens. J Environ Pathol Toxicol Oncol 13: 191-197, 1994.

15 Femia AP, Caderni G, Ianni M, Salvadori M, Schijlen E, Collins G, Bovy A and Dolara P: Effect of diets fortified with tomatoes or onions with variable quercetin-glycoside content on azoxymethane-induced aberrant crypt foci in the colon of rats. Eur J Nutr 42: 346-352, 2003.

16 Wright $\mathrm{N}$ : Epithelial stem cell repertoire in the gut; clues to the origin of cell lineages, proliferative units and cancer Int J Exp Pathol 81: 117-143, 2000.

17 Rubio CA: Putative stem cells in mucosas of the esophagogastrointestinal tract. Chapter 10. In: Stem Cell, Regenerative Medicine and Cancer. Singh SR (ed.). Haupauge, NY, USA: Nova Science Publishers, Inc. pp. 279-308, 2011.

18 Bird RP, McLellan EA and Bruce WR: Aberrant crypts, putative precancerous lesions, in the study of the role of diet in the aetiology of colon cancer. Cancer Surv 8: 189-200, 1989.

19 Ghirardi M, Nascimbeni R, Villanacci V, Fontana MG, Di Betta E and Salerni B: Azoxymethane-induced aberrant crypt foci and colorectal tumors in F344 rats: sequential analysis of growth. Eur Surg Res 31: 272-280, 1999.

20 Ochiai M, Hippo Y, Izumiya M, Watanabe M and Nakagama H: Newly defined aberrant crypt foci as a marker for dysplasia in the rat colon. Cancer Sci 105: 943-950, 2014.

21 St Clair WH and Osborne JW: Crypt fission and crypt number in the small and large bowel of postnatal rats. Cell Tissue Kinet 18: 255-262, 1985.

22 Tan CW, Hirokawa Y, Gardiner BS, Smith DW and Burgess AW: Colon cryptogenesis: asymmetric budding. PLoS One 8: e78519, 2017.

23 Boman BM and Fields JZ: An APC:WNT countercurrent-like mechanism regulates cell division along the human colonic crypt axis: a mechanism that explains how APC mutations induce proliferative abnormalities that drive colon cancer development. Front Oncol 3: 244-250, 2013.

24 Shih IM, Wang TL, Traverso G, Romans K, Hamilton SR, BenSasson S, Kinzler KW and Vogelstein B: Top-down morphogenesis of colorectal tumors. Proc Natl Acad Sci USA 98: 2640-2545, 2001. 